\title{
Biblioguías en línea en las bibliotecas universitarias españolas
}

Online biblioguides in Spanish academic libraries

\author{
José Luis HeRrera MORILLAS \\ Facultad de Ciencias de la Documentación y la Comunicación, Universidad de Extremadura, \\ Plazuela de Ibn Marwan, s/n, ES-06001 Badajoz (España), jlhermor@unex.es
}

\begin{abstract}
Resumen
Se inventarían y analizan las secciones de biblioguías de las bibliotecas de las universidades españolas que están disponibles en sus sitios webs. Se estudió la totalidad de las universidades españolas, con resultados positivos en 41 bibliotecas. Para cada guía se identificó su nombre, URL, ubicación, concepto, organización y clasificación, y formatos. Las biblioguías se conciben como una manifestación de la formación de usuarios en el ámbito de alfabetización informacional en un entorno en línea
\end{abstract}

Palabras clave: Biblioguías. Formación de usuarios. Formación en línea. Alfabetización informacional. Bibliotecas universitarias. España.

\section{Introducción}

Nos ha parecido interesante estudiar los apartados o secciones que algunas bibliotecas universitarias presentan en sus sitios webs para alojar biblioguías, después de haber comprobado su existencia y utilidad como usuario en el ejercicio de nuestra actividad de docente universitario. También porque, como investigador en el ámbito de las ciencias de la información y la documentación, nos ha interesado analizarlas en relación con los cambios acaecidos en las bibliotecas universitarias en los últimos tiempos.

Llevamos ya tiempo presenciando modificaciones en las actividades y funciones de las bibliotecas universitarias que han dado lugar a "una recreación de las mismas" definiendo nuevas funciones como la "alfabetización informacional, bibliotecas digitales y preservación de documentos electrónicos" (Iribarren-Maestro et al., 2015, p. 131).

Las bibliotecas universitarias españolas han ampliado su clásico rol de apoyo a la docencia, aprendizaje e investigación con otros en los que se incluyen el aprendizaje sobre la localización, selección, evaluación, reelaboración, uso y comunicación de la información. Es decir la contribución a la formación de la competencia infor-

\begin{abstract}
The sections for biblioguides of all the Spanish university library websites were analysed and described. 41 of them have online biblioguide sections. They were described by their name, URL, location, concept, organization and classification, and formats. Library biblioguides are conceived as a manifestation of user training in the field of information literacy in online environments.
\end{abstract}

Keywords: Biblioguides. User training. Online education. Information literacy. Academic libraries. Spain.

macional. Así lo expone Gómez Hernández (2010, p. 39):

\begin{abstract}
Las bibliotecas se interesan por las competencias informacionales porque actualmente son, antes que una colección física y electrónica en unos espacios virtuales o reales, un lugar donde un conjunto de profesionales aspiran a asegurarse de que los estudiantes aprendan, para hacerles competentes en las habilidades digitales e informacionales mientras estén en la universidad y a lo largo de la vida.
\end{abstract}

En el siglo XXI los servicios de la biblioteca universitaria se han ampliado y diversificado para seguir atendiendo de manera eficaz las demandas de los usuarios, entre las que están enseñarles a sacar provecho de la gran cantidad de información disponible (Serrano Vicente, 2005); también, por el mayor uso de la biblioteca en el entorno virtual, que ha llevado a las bibliotecas a consolidar el empleo de sus sitios webs, por ejemplo, ofertando materiales formativos como es el caso de la biblioguías objeto de este trabajo, concebidas como una vía de formación de usuarios para que puedan llegar a ser autónomos en el uso de los recursos y los servicios que la biblioteca ofrece (Pérez Hidalgo y Méndez Martínez, 2000).

Una de las cualidades que se le piden en la actualidad a los servicios bibliotecarios en el contexto universitario es que sean proactivos "asumiendo que la era del usuario presencial y 
físico es una realidad en retroceso" (Pinto, Sales, y Osorio, 2008, p. 107). De este modo las bibliotecas universitarias se mantendrán vivas, y no desperdiciarán las oportunidades que se presentan en el nuevo entorno (Anglada, 2012, p. 556):

En plena efervescencia de las disrupciones que tienen lugar el mundo digital, las bibliotecas universitarias han sabido hacer evolucionar los servicios tradicionales a la vez que, cabalgando las tecnologías, han creado nuevos servicios siguiendo la estela de las necesidades en parte tradicionales, en parte cambiantes, de sus usuarios.

Las biblioguías que estudiamos en este trabajo son un material online que se puede enmarcar dentro de las iniciativas sobre formación de usuarios para facilitar una utilización más eficaz de los recursos relacionados con la gestión de la información y los servicios que la biblioteca ofrece.

\subsection{Objetivos}

El objetivo que se pretende con este trabajo es identificar las bibliotecas universitarias que cuentan con secciones de biblioguías, disponibles en sus sitios webs. Este objetivo se desglosa en cinco más específicos: diseñar una lista de análisis para la recogida de datos; comprobar la difusión y alcance de los sitios webs como herramienta de trabajo; observar la relación de las biblioguías con la formación de usuarios en un contexto online; abordar las biblioguías en relación con la formación en alfabetización informacional en un entorno virtual; y delimitar el concepto de biblioguía a partir del análisis realizado.

\subsection{Formación de usuarios}

En nuestros días la formación de usuarios es fruto de la evolución de una mera instrucción bibliográfica a una nueva modalidad vinculada a la alfabetización informacional con el propósito de conseguir las habilidades necesarias para localizar y utilizar la información pertinente.

Las publicaciones que abordan este planteamiento de la formación de usuarios son abundantes. Por ello hacemos una breve selección comenzando por las realizadas fuera de España. Sobre el papel de instrucción de los bibliotecarios y su evolución hacia la necesidad de afrontar los recursos de instrucción en línea destacamos el trabajo de Dewald (1999) donde se analizan buenas prácticas y criterios que puedan servir de utilidad a las bibliotecarios para elaborar tutoriales online; y el de Warnken (2004) que, desde el objetivo de estudiar el caso las bibliotecas académicas de Rumanía, aborda los métodos y recursos que se deben utilizar en la instrucción de la alfabetización informacional impartida por este tipo de bibliotecas, partiendo de que la alfabetización informacional es muy importante para los usuarios académicos por sus implicaciones en la práctica profesional dentro de cualquier ámbito.

Yi (2005) justifica esta demanda en el quehacer de los bibliotecarios por el auge de la biblioteca electrónica y la necesidad de contribuir a mejorar la vida educativa de los alumnos mediante servicios en línea. También, es necesario facilitar a los usuarios las habilidades necesarias para reconocer el valor de los recursos de la biblioteca y para localizar y usar estos recursos con éxito (Bracke y Dickstein, 2002). Sacchanand y Jaroenpuntaruk (2006) han explicado la importancia del aprendizaje autodirigido en el contexto de la biblioteca, donde la enseñanza basada en la web permite a los individuos aprender por sí mismos, fomentando a la vez la auto-reflexión y el aprendizaje activo.

Los tutoriales web como herramientas para formar en el uso de los recursos de información son frecuentes en las bibliotecas y existen también estudios como el de Tobin y Kesselman (1999) o el Fernández-Ramos (2015) que abordan cuestiones sobre el diseño de los tutoriales web para la formación de usuarios virtuales. En este ámbito destacan Somoza-Fernández y Rodríguez-Parada (2011) que diseñan un método de evaluación de tutoriales web ilustrado con ejemplos de buenas prácticas de bibliotecas universitarias. En otros trabajos se analizan las aportaciones de bibliotecas concretas como el de Rutter y Matthews (2002) dedicado a la Universidad de Bournemouth y su tutorial "InfoSkiIIs". Giappiconi et al. (2001) recopilan y analizan ejemplos destacados de bibliotecas públicas de todo el mundo.

Otro aspecto sobre el que se ha investigado es el de la ayuda en línea: Wickramanayake (2012) estudia las aplicaciones de instrucción y las herramientas de ayuda que utilizan las bibliotecas académicas en sus sitos webs, como contexto para el estudio detallado del caso de las bibliotecas académicas de Sri Lanka. Fernández-Ramos (2016) evalúa la presencia de tutoriales en línea en los sitios webs de las bibliotecas de universidades públicas en México, analizando sus principales características. Zhang, Stonebraker y Promann (2016) examinan las preferencias y expectativas de los usuarios de los canales de ayuda de la biblioteca en general y de la ayuda en línea en particular mediante una encuesta sobre sus preferencias y expectativas de ayuda en línea, formato de contenido y canales generales de ayuda. 
Terminamos la cita de trabajos de ámbito internacional con dos de carácter bibliográfico y que son fuentes de información actualizada sobre recursos de alfabetización informacional y formación de usuarios, el de Detmering et al. (2015) y el de Reynolds et al. (2016), que proporcionan un resumen introductorio y una bibliografía anotada de publicaciones (artículos, monografías, ponencia, etc.) que abarcan todo tipo de bibliotecas.

Autores de nuestro país también han estudiado la formación de usuarios. En particular, las siguientes aportaciones nos parecen significativas y apropiadas para enmarcar el trabajo que presentamos. Gómez Hernández y Benito Morales (2001) trazan de una manera sintética y clara la evolución de la formación de usuarios desde su concepción tradicional hasta la alfabetización informacional. Gómez Hernández (2005) precisa que la alfabetización informacional se propone alcanzar competencias no trabajadas habitualmente en la formación de usuarios (evaluación de los recursos, comprensión, utilización y comunicación de la información) y ha comprobado (Gómez Hernández, 2010) cómo actualmente todas las bibliotecas tienen programas de alfabetización informacional.

Martínez Ballester (2014) realiza el estado de la cuestión más reciente publicado sobre la formación de usuarios en línea en las bibliotecas universitarias, centrándose sobre todo en el análisis de los programas de formación.

Para finalizar citamos el trabajo de López Zazo (2007) sobre planificación de la formación usuarios para un CRAI donde se puede consultar abundante bibliografía en relación con la formación de usuarios en la bibliotecas universitarias.

\subsection{Biblioguías}

Se puede considerar que las biblioguías son una manifestación del fomento del autoaprendizaje favorecido en el entorno de la biblioteca híbrida por los programas de alfabetización informacional y educación documental, como afirman Pinto, Sales y Osorio (2008):

La presencia creciente de las tecnologías de información y comunicación en los entornos de educación superior, así como su impacto en la docencia semipresencial y virtual, justifica que el futuro CRAI colabore en el diseño y desarrollo de herramientas didácticas y educativas para fomentar la autonomía de profesores y estudiantes.

La biblioteca de la Universidad de Burgos define así las biblioguías (Véase la Tabla 4):

Material de apoyo para facilitar el uso de los recursos de información a través del autoaprendizaje (elaboración de trabajos fin de grado y máster, gestión de referencias bibliográficas y citas, edición y producción científica...).

Respecto a la existencia de estudios previos sobre este tipo de guías no nos consta que se hayan realizado abordándolas de manera exclusiva. Por ello, sólo contamos dentro de la ya mencionada bibliografía sobre formación de usuarios y/o alfabetización informacional en el ámbito de las bibliotecas universitarias con alusiones a materiales y aspectos que guardan cierta relación con las guías objeto de nuestro estudio. A continuación indicamos algunas en orden cronológico de publicación.

En el trabajo de Somoza-Fernández (2003) sobre tutoriales temáticos se menciona una clasificación de los principales métodos de enseñanza utilizados a través de la web en las bibliotecas universitarias, en los que se incluyen: a) guías de uso y manuales; b) demostraciones; y c) tutoriales.

Somoza-Fernández y Abadal (2007) ven en el fomento de la autoformación y la creación de materiales y experiencias de formación virtual una manifestación de una formación más individualizada que refleja la evolución de la formación de usuarios tradicional hacia a un entorno más en concordancia con la alfabetización informacional.

La obra de Pinto, Sales y Osorio (2008) apoya el fomento del autoaprendizaje por parte de las bibliotecas universitarias mediante la elaboración, el diseño y desarrollo de herramientas didácticas y educativas para fomentar la autonomía de profesores y estudiantes, en las que pueden enmarcarse las biblioguías.

El trabajo de Gómez Hernández (2010) menciona la publicación de guías explicativas como una de las tareas clásicas de formación de usuarios y la elaboración los tutoriales online, como práctica más reciente, dirigida hacia la alfabetización informacional.

Wickramanayake (2012) defiende que los bibliotecarios deben preparar tutoriales y materiales de instrucción en línea y deberán integrarlos racionalmente en los sitios webs junto con las herramientas de ayuda. Estos recursos han sido estudiados por Fernández-Ramos (2016) comprobando que la mayoría de los tutoriales se ocupan del uso de los servicios de biblioteca o de la gestión de un recurso de información específico. Los formatos más habituales son pdf y video, y generalmente tienen pocos elementos de aprendizaje activo.

Martínez Ballester (2014) relaciona las guías y manuales como uno de los métodos utilizados 
para ofrecer formación de usuarios en la modalidad online en las bibliotecas universitarias.

Fernández-Ramos (2015) hace hincapié en que la elaboración de estos materiales está acaparando cada vez más interés, pero su elaboración no siempre es sencilla y requiere tiempo y dinero para evitar productos que simplemente dupliquen los materiales impresos utilizados con anterioridad, por el contrario se deben aprovechar las posibilidades tecnológicas e incorporarlas en su diseño.

Tovar-Sanz (2015) en su estudio sobre las secciones de apoyo a la investigación en los sitios webs de las bibliotecas universitarias españolas dedica un apartado a la formación de usuarios y concluye sobre el empleo de tutoriales que:

El $88 \%$ de las bibliotecas universitarias españolas cuelgan tutoriales en sus webs. Estos materiales pueden ser consultados en cualquier momento y lugar, el servicio se optimiza y se ahorra el tiempo que se invertiría en formaciones grupales y/o entrevistas personales. Las temáticas más comunes son la descripción básica de la biblioteca y sus servicios, la búsqueda en el catálogo, el uso de las bases de datos, el uso de los gestores de referencia, información sobre cómo citar y contra el plagio.

\section{Metodología}

La primera tarea consistió en localizar los sitios webs de las bibliotecas universitarias españolas. Para localizar las bibliotecas universitarias se ha utilizado los recursos Directorio bibliotecas españolas (2017) y Anexo: Universidades de España (2017). Se han visitado todas las universidades (públicas, privadas, presenciales, online, etc.), en total 83 universidades. A continuación se procedió a la exploración de los sitios webs para comprobar cuáles cuentan con secciones para biblioguías. Seguidamente se realizó el análisis de estas secciones o apartados. Para ello se elaboró un modelo o lista que consta de seis elementos (Tabla I).

\begin{tabular}{ll}
\hline Nombre & $\begin{array}{l}\text { Expresión utilizada para denominar la } \\
\text { sección de biblioguias }\end{array}$ \\
\hline URL & Localización del recurso en Internet \\
\hline Ubicación & $\begin{array}{l}\text { Lugar exacto donde se localiza la sección } \\
\text { de las biblioguias en la web de la biblioteca }\end{array}$ \\
\hline Concepto & $\begin{array}{l}\text { Cuando aparece, se reproduce la } \\
\text { definición o descripción que la biblioteca } \\
\text { realiza de este tipo de recurso }\end{array}$ \\
\hline $\begin{array}{l}\text { Organización y } \\
\text { clasificación }\end{array}$ & $\begin{array}{l}\text { Forma y estructura para organizar y } \\
\text { presentar las guías }\end{array}$ \\
\hline Formatos & $\begin{array}{l}\text { Formatos que se emplean en la } \\
\text { elaboración de las biblioguías }\end{array}$ \\
\hline
\end{tabular}

Tabla I. Lista de análisis

Sólo se seleccionaron las bibliotecas que cuentan con secciones o apartados específicos para este tipo de guías, por lo tanto no se tuvieron en cuenta las bibliotecas con guías que responden a las características de las analizadas ubicadas en otros apartados o secciones.

Con los datos de cada biblioteca se elaboraron fichas que se adjuntan en el anexo final, en el que las bibliotecas aparecen ordenadas por comunidades autónomas. La revisión final de los datos para la elaboración de este estudio tuvo lugar en marzo de 2017. Sólo se seleccionaron las bibliotecas que cuentan con secciones o apartados específicos para este tipo de guías, por lo tanto no se tuvieron en cuenta las bibliotecas con guías que responden a las características de las analizadas que estuvieran ubicadas en otros apartados o secciones.

\section{Resultados}

De las 83 universidades analizadas, 41 bibliotecas universitarias $(49,3 \%)$ cuentan con secciones específicas para las biblioguías (tabla II, en apéndice). De estas 41 bibliotecas 4 incluyen 2 secciones para las biblioguías. Esto hace que el conjunto de secciones analizadas sea de 45.

La sección de biblioguías es un recurso ampliamente implantado en las bibliotecas universitarias españolas. Hay que matizar que entre las bibliotecas analizadas se encuentran algunas que por sus características peculiares pueden justificar que no incluyan secciones para biblioguías; nos referimos a las universidades online, a las eclesiásticas o a las internacionales, que se alejan del modelo estándar de universidad. También, se debe tener en cuenta que hay bibliotecas que no tienen secciones específicas para biblioguías, pero incluyen algunas en otras secciones, sobre todo en las de Apoyo a la investigación. Esta sección es muy frecuente en nuestras bibliotecas universitarias y ha sido objeto de varios estudios (Tovar-Sanz, 2015; Iribarren-Maestro et al., 2015).

Respecto a los nombres que se utilizan para denominar a las secciones que albergan las biblioguías (Tabla II) los datos obtenidos son los siguientes: 11 bibliotecas las engloban con los tutoriales ("Guías y tutoriales"; "Tutoriales de la biblioteca", etc.); 8 bibliotecas emplean la palabra guía, sola o junto a otro calificativo ("Guías rápidas"; "Guías de la biblioteca", etc.); 7 bibliotecas utilizan expresiones relacionadas con autoformación o la formación ("Guías y formación"; "Material de formación"; "Autoformación", etc.); 7 bibliotecas usan la denominación de guías temáticas; 6 bibliotecas han optado por vincular este tipo de guías con la ayuda ("Guías y ayuda"; "Guías de ayuda"; "Ayuda", etc.); y 6 bibliotecas han elegido la denominación de bi- 
blioguías. También, hay que tener en cuenta que 4 bibliotecas incluyen dos secciones para las guías con denominaciones diferentes. Son las bibliotecas de las universidades de Alcalá de Henares ("Guías temáticas"; "Ayuda”); Carlos III
("Tutoriales y guías"; "Guías temáticas”); Jaime I ("Materiales de formación"; "Guías y manuales") y Las Islas Baleares ("Ayuda"; "Guías temáticas").

\begin{tabular}{|c|c|c|}
\hline Universidad & Sección & URL \\
\hline \multirow[t]{2}{*}{ Alcalá de Henares } & Guías temáticas & http://wikibuah.pbworks.com/w/page/65722818/Inicio-Gu\%C3\%83\%C2\%ADas \\
\hline & Ayuda & http://biblioteca.uah.es/servicios/accesibilidad.asp \\
\hline Alicante & Guías temáticas & https://biblioteca.ua.es/es/encuentra-informacion/guias-tematicas/guias-tematicas.html \\
\hline Almería & Guías temáticas & http://cms.ual.es/UAL/universidad/serviciosgenerales/biblioteca/servicios/FOR_24_GUIAS \\
\hline Autónoma de Barcelona & Guías de las bibliotecas de la UAB & http://ddd.uab.cat/collection/guibib?ln=es \\
\hline Autónoma de Madrid & Biblioguías de la Biblioteca & http://biblioguias.uam.es/c.php?g=235585 \\
\hline Burgos & Autoformación & http://wwww.ubu.es/aprendizaje-e-investigacion/aulabierta-formacion-guias/autoformacion \\
\hline Cantabria & Guías y tutoriales & http://www.buc.unican.es/formacion \\
\hline \multirow[t]{2}{*}{ Carlos III } & Tutoriales y guías & $\begin{array}{l}\text { http://www.uc3m.es/ss/Satellite/Biblioteca/es/TextoMixta/1371212366749/UTEID_Unidad_de_ } \\
\text { Tecnologia_Educativa_e_Innovacion_Docente }\end{array}$ \\
\hline & Guías temáticas & http://uc3m.libguides.com/guias_tematicas \\
\hline Castilla-La Mancha & Tutoriales de la biblioteca & http://biblioteca.uclm.es/tutoriales.html \\
\hline Católica de San Antonio & Videos formación biblioteca & http://biblioteca.ucam.edu/servicios/gabinete-formacion/videos-formacion/formacion \\
\hline Complutense de Madrid & Autoformación & https://biblioteca.ucm.es/autoformacion \\
\hline Córdoba & Guías y tutoriales BUCO & http://www.uco.es/servicios/biblioteca/formacion/ayestudio.html \\
\hline Deusto & Biblioguías & http://biblioguias.biblioteca.deusto.es/ \\
\hline Extremadura & Biblioguías & http://biblioguias.unex.es/ \\
\hline Granada & Guías temáticas & http://biblioteca.ugr.es/pages/biblioteca_electronica/guias_tematicas \\
\hline Huelva & Guías y ayuda & http://guiasbuh.uhu.es/guias \\
\hline \multirow[t]{2}{*}{ Islas Baleares } & Ayuda & http://biblioteca.uib.cat/oferta/ajuda/ \\
\hline & Guías temáticas & http://biblioteca.uib.cat/oferta/recursos_electronics/guies_tematiques/ \\
\hline \multirow[t]{2}{*}{ Jaime I } & Material de formación & http://www.uji.es/serveis/cd/bib/serveis/investigacio/materialformacio/ \\
\hline & Guías y manuales & http://repositori.uji.es/xmlui/handle/10234/10776 \\
\hline La Coruña & Guías & http://www.udc.es/biblioteca/servizos/apoio_investigacion/recursos_info/index2.html \\
\hline La Laguna & Guías y tutoriales & https://www.ull.es/view/institucional/bbtk/Guias_y_tutoriales/es \\
\hline Las Palmas de Gran Canaria & Biblioguías & http://biblioguias.ulpgc.es/ \\
\hline León & Guías rápidas & http://biblioteca.unileon.es/content/gu\%C3\%ADas-r\%C3\%A1pidas \\
\hline Lérida & BiblioGuías & http://biblioguies.udl.cat/friendly.php?s=guies \\
\hline Málaga & Guías y tutoriales & http://www.uma.es/ficha.php?id=74832 \\
\hline Murcia & Autoformación & http://www.um.es/web/biblioteca/contenido/formacion/autoformacion \\
\hline Navarra & Recursos por materias y guías & http://apps-bibl.si.unav.es/sp/subjects/index.php \\
\hline Oviedo & Ayudas & https://buo.uniovi.es/ayudas \\
\hline Politécnica de Cartagena & Guías y manuales & http://www.bib.upct.es/guias-manuales \\
\hline Politécnica de Valencia & Biblioguías & http://biblioguias.webs.upv.es/bg/index.php/es/ \\
\hline Pompeu Fabra & Guías y tutoriales & https://www.upf.edu/bibtic/guiesiajudes/ \\
\hline Pontificia de Comillas & Guías y formación & http://www.comillas.edu/es/biblioteca/guias-y-tutoriales \\
\hline Pontificia de Salamanca & Guías de autoformación & https://www.upsa.es/biblioteca/servicio/tutoriales/autoformacion.php \\
\hline Pública de Navarra & Guías temáticas & http://guias-tematicas.unavarra.es/sp/subjects/index.php \\
\hline Rey Juan Carlos & Guías y tutoriales & http://www.urjc.es/estudiar-en-la-urjc/biblioteca/400-formacion \\
\hline Rovira i Virgili & Guías y tutoriales & $\begin{array}{l}\text { http://www.urv.cat/ca/vida-campus/serveis/crai/que-us-oferim/formacio-competencies- } \\
\text { nuclears/autoformacio/ }\end{array}$ \\
\hline Santiago de Compostela & Guías & http://sp.bugalicia.org/san/subjects/index.php \\
\hline Sevilla & Otras guías y ayudas & http://guiasbus.us.es/c.php?g=129439\&p=845019 \\
\hline UNED & Guías y tutoriales & http://portal.uned.es/portal/page?_pageid=93,26044627\&_dad=portal\&_schema=PORTAL \\
\hline Valladolid & Guías rápidas & $\begin{array}{l}\text { http://biblioteca.uva.es/export/sites/biblioteca/3.servicios/3.09.labiblioteca } \\
\text { conlainvestigacion/3.09.03.guiasrapidas/index.html }\end{array}$ \\
\hline Vigo & Guías y tutoriales & http://sp.bugalicia.org/vig/subjects/index.php \\
\hline Zaragoza & Guías de ayuda & http://biblioteca.unizar.es/ayuda-y-formacion/guias-de-ayuda \\
\hline
\end{tabular}

Tabla II. Secciones de Biblioguías en las bibliotecas universitarias españolas.

Aunque la expresión biblioguía no la utilizan todas las bibliotecas nos parece la más adecuada y original, pues identifica bien el producto: guías elaboradas por la biblioteca y relacionadas con la gestión bibliográfica y la gestión de la información. Por ello la hemos utilizado para el título de este trabajo. En este estudio también nos hemos fijado en la ubicación que ocupan las secciones de las biblioguías en los sitios webs de las bibliotecas (véase el Apéndice), pues de ello depende que los usuarios puedan encontrarlas de la manera más intuitiva posible.

De los 45 sitios analizados, en 6 sitios (13,3\%) la sección de biblioguías se localiza en un apartado principal, de primer nivel, en el sitio web de 
la biblioteca. Lo más frecuente es que se incluyan en un subapartado, de segundo nivel, es el caso de 27 secciones de biblioguías (60\%). En la Tabla III se indican las denominaciones de los apartados principales en las que se incluyen. Se observa una gran variedad de denominaciones: 22 denominaciones diferentes. Las 11 secciones de biblioguías restantes $(24,4 \%)$ están localizadas en apartados de tercer nivel.

Sobre el concepto, en 18 bibliotecas (43,9\%) hemos localizado algún tipo de mención sobre las biblioguías que nos parece interesante recoger porque ayuda a deducir la función con la que se conciben (Tabla IV).

La lectura detenida de estas menciones nos llevan a agruparlas en 4 categorías teniendo en cuenta los aspectos en los que más incidencia se hace:

- La agrupación por áreas temáticas (5): Alcalá de Henares, Almería, Granada, Navarra, Politécnica de Valencia.

- La formación en recursos y servicios de la biblioteca (5): Autónoma de Barcelona, Deusto, Murcia, Rovira i Virgili, Zaragoza.

- El uso y búsqueda de información (5): Burgos, Cantabria, Huelva, Las Islas Baleares, León.

- El apoyo a la docencia e investigación (2): Autónoma de Barcelona, Lérida.
El texto de la Universidad de Oviedo destaca por igual tanto la formación en recursos y servicios de de la biblioteca, como el apoyo a la docencia e investigación.

\begin{tabular}{ll}
\hline Denominación & $N^{\circ}$ \\
\hline Aprendizaje e investigación & 3 \\
\hline Ayudas y formación & 1 \\
\hline Ayudas y tutoriales & 1 \\
\hline Biblioteca electrónica & 1 \\
\hline Biblioteca & 1 \\
\hline Colecciones y recursos & 1 \\
\hline Cómo usar la biblioteca & 1 \\
\hline Cursos de formación & 1 \\
\hline Encontrar información & 1 \\
\hline Encuentra información & 1 \\
\hline Estudia e investiga & 1 \\
\hline Formación de usuarios & 2 \\
\hline Formación & 3 \\
\hline Investigación y aprendizaje & 1 \\
\hline Qué ofrecemos & 1 \\
\hline Recursos electrónicos & 1 \\
\hline Recursos de información & 1 \\
\hline Servicios & 2 \\
\hline Soporte a la formación & 1 \\
\hline Tu biblioteca & 1 \\
\hline Tutoriales & 1 \\
\hline Utilizar & 1 \\
\hline
\end{tabular}

Tabla III. Denominaciones en apartados principales

\begin{tabular}{|c|c|}
\hline Universidad & Definición \\
\hline $\begin{array}{l}\text { Alcalá } \\
\text { de Henares }\end{array}$ & "La Biblioteca estructura por materias la información, lo que facilita conocer sus recursos, dónde y cómo localizarlos" \\
\hline Almería & "Recursos de información disponibles en la Biblioteca, agrupados por áreas temáticas" \\
\hline $\begin{array}{l}\text { Autónoma } \\
\text { de } \\
\text { Barcelona }\end{array}$ & $\begin{array}{l}\text { "Engloba todo tipo de guías enfocadas al conocimiento de las herramientas para la investigación, las bases de datos y los } \\
\text { recursos temáticos que la UAB pone a vuestra disposición" }\end{array}$ \\
\hline $\begin{array}{l}\text { Autónoma } \\
\text { de Madrid }\end{array}$ & "Guías, tutoriales y recursos de apoyo para la investigación y la docencia" \\
\hline Burgos & $\begin{array}{l}\text { "Material de apoyo para facilitar el uso de los recursos de información a través del autoaprendizaje (elaboración de trabajos } \\
\text { fin de grado y máster, gestión de referencias bibliográficas y citas, edición y producción científica...)" }\end{array}$ \\
\hline Cantabria & "Guías, tutoriales y materiales formativos que cubren diversos aspectos relacionados con el uso de la información científica" \\
\hline Deusto & "Te orientan sobre los recursos y servicios más relevantes de la biblioteca" \\
\hline Granada & $\begin{array}{l}\text { "Una ayuda que pretende organizar diferentes recursos electrónicos, no en función del formato, soporte o medio de acceso, } \\
\text { sino agrupados por amplias áreas temáticas" }\end{array}$ \\
\hline Huelva & "Colección de recursos clasificados por temas que te orientarán a la hora de buscar la información que necesitas" \\
\hline $\begin{array}{l}\text { Islas } \\
\text { Baleares }\end{array}$ & $\begin{array}{l}\text { "Selecció de recursos d'informació en diferents àmbits temàtics de suport a la docència, l'aprenentatge i la investigació. } \\
\text { Tenen com a objectiu ajudar i orientar en la recerca d'informació sobre una àrea temàtica concreta" }\end{array}$ \\
\hline León & "Intenta solventar necesidades de profesores y alumnos según dos cuestiones ¿Qué necesito? Y ¿Dónde lo busco?” \\
\hline Lérida & $\begin{array}{l}\text { "Selecció de recursos d'informació vinculats amb l'aprenentatge, la docència i la recerca. Contenen recursos web rellevants i } \\
\text { l'accés als recursos electrònics més significatius per a cada àmbit de coneixement" }\end{array}$ \\
\hline Murcia & $\begin{array}{l}\text { "Guías de uso de los recursos más utilizados. En algunos casos son tutoriales que ofrecen los creadores de los recursos de } \\
\text { información. Otras son guías y presentaciones realizadas por bibliotecarios de la Universidad de Murcia, utilizadas en } \\
\text { nuestras sesiones de formación" }\end{array}$ \\
\hline Navarra & "Guías elaboradas por los bibliotecarios temáticos con los recursos más interesantes en cada área" \\
\hline Oviedo & $\begin{array}{l}\text { "Información que necesitas para utilizar nuestros recursos, herramientas y servicios (guías, tutoriales, manuales, etc.) y para } \\
\text { desarrollar tu actividad académica a través de nuestros minisites que contienen fuentes de información para alumnos de }\end{array}$ \\
\hline
\end{tabular}


grado, alumnos de postgrado, investigadores y diversas guías temáticas"

\begin{tabular}{ll}
\hline $\begin{array}{l}\text { Politécnica } \\
\text { de Valencia }\end{array}$ & "Guías y ayudas donde encontrarás diferentes recursos elaborados o recopilados por las Bibliotecas UPV, organizados de \\
\hline Rovira i & "Materials d'autoaprenentatge amb la finalitat que pugueu aprendre de manera autònoma el funcionament del catàleg, de \\
Virgili & les bases de dades, dels serveis o dels recursos d'informació que posem al vostre abast des del CRAl" \\
\hline Zaragoza & "La BUZ ha creado diverso material de ayuda para la formación en sus recursos"
\end{tabular}

Tabla IV. Concepto de biblioguías

Los datos sobre la organización y clasificación de las biblioguías evidencian la diversificación en las formas de agruparlas y presentarlas (véase el Apéndice). En primer lugar, hay que distinguir entre las bibliotecas con secciones que sólo incluyen guías temáticas, que se limitan a agruparlas en varias materias, y el resto de bibliotecas, que ofrecen una gran diversidad a la hora de agrupar las guías; con sistematizaciones muy variadas en cuanto a la extensión y expresiones que utilizan, que sin embargo no presentan tanta diversidad en relación a la temátìca y contenidos de las guías.

Una vez analizadas las secciones donde se presentan las biblioguías y las formas de organizarlas, se pueden establecer cinco modelos:

- Atendiendo a la finalidad de las guías, utilizan categorías del tipo: "Guías para elaborar un trabajo científico", "Estudia e investiga", "Ayudas para utilizar gestores de citas y elaborar bibliografía", "Apoyo al aprendizaje", "Autoformación", etc.

- Atendiendo a la utilización de servicios y recursos de la biblioteca que las guías pretenden enseñar, utilizan categorías del tipo: "Consulta al catálogo", "Consulta a los recursos electrónicos", "Conoce la biblioteca", "Renovación del material bibliográfico", "Conócenos", "Ayudas para utilizar bases de datos", etc.

- Atendiendo al alcance de la guías, utilizan categorías del tipo: "Guías generales", "Guías genéricas", "Guías rápidas", "Guías de recursos", "Guías de uso general", "Guías temáticas", "Para el aprendizaje", "Guías por bibliotecas", "Formación básica”, "Formación especializada", etc.

- Atendiendo al formato de la guías, utilizan categorías del tipo: "Guías en Slideshare", "Guías en ISSUU”, "Videotutoriales”, etc.

- Atendiendo a los destinatarios, utilizan categorías del tipo: "Personal docente e investigador", "Estudiantes de grado", "Estudiantes de Máster, postgrado y doctorado", "Alumnos de primer año", etc.
Los resultados del análisis de los formatos en los que se presentan las biblioguías nos indican que de las 45 secciones recopiladas, 24 (53,3 $\%$ ) presentan las biblioguías en único formato (18 en html, 4 en pdf y 2 en vídeo). En los 21 sitios restantes $(47,7 \%)$ se utilizan más de un formato. Los más frecuentes son html, pdf y video. Otros formatos utilizados son Ppt, en 5 bibliotecas; Prezi, en 3 bibliotecas; SlideShare, en 3 bibliotecas; e Issuu, en 3 bibliotecas.

Respecto al diseño formal que presentan las biblioguías existe cierta variedad. Comentamos los modelos más significativos:

- Empleo del formato html con un diseño uniforme preestablecido, en el que junto al texto estructurado en apartados y subapartados (a veces redactado con interrogantes que se van respondiendo) se insertan enlaces, gráficos, imágenes, archivos pdf, ficheros adjuntos, videos (ejemplos: Burgos, Cantabria, Deusto, Extremadura, Huelva, Las Palmas de Gran Canaria, Lérida, Santiago de Compostela o Sevilla). Es el modelo más extendido.

- Documentos en pdf, bien fruto de la reproducción en este formato de los folletos o dípticos editados en papel; o bien, de documentos elaborados expresamente en este formato con un diseño uniforme en cuanto al tipo letra, colores, encabezado, etc. Dentro de los documentos que siguen esta segunda opción diferenciamos entre los que siguen una estructura académica de documento de estudio (ejemplos: Carlos III, Islas Baleares); y los que han sido elaborados de forma más esquemática y pedagógica (ejemplos: Autónoma de Barcelona, Pontificia de Salamanca).

- Recopilación de una variedad de documentos en diferentes formatos con falta de uniformidad: por ejemplo, documentos diseñados en ppt pero impresos en pdf; documentos de texto de diferentes diseños en formato pdf; folletos y dípticos en versión pdf (ejemplos: Córdoba, León, Zaragoza).

- Agrupación de material diverso que se ha utilizado en cursos o disponible en otras secciones de la web. Por ello, se aprecia que no han sido diseñados expresamente para esta 
sección y hay falta de uniformidad (ejemplo: Complutense).

- Página que facilita el acceso a documentos de diferente procedencia, años de publicación, diseños, etc. a modo de repositorio (ejemplos: La Coruña, Jaime I).

- Material de elaboración propia con un diseño, estilo y estructura uniforme preestablecidos; junto a otros recursos de procedencia externa (ejemplo: Murcia).

\section{Conclusiones}

Este trabajo nos ha permitido comprobar cómo las bibliotecas universitarias han consolidado el empleo de los sitios webs como un espacio y herramienta de trabajo imprescindible, en el que prestan servicios integrados en el contexto virtual, que les ha catapultado a una nueva dimensión y una mayor visibilidad. Servicios que todavía se combinan con su prestación en el contexto presencial, característica del modelo de biblioteca híbrida en el que todavía nos encontramos. Esta nueva dimensión ha afectado a uno de los servicios bibliotecarias más clásicos y característicos: la formación de usuarios.

Es un hecho que Internet se ha convertido en la principal fuente de búsqueda por parte de los alumnos y docentes para recopilar información y documentos. Lo cual supone que la biblioteca universitaria tiene que competir con esta realidad. En este contexto la principal arma con la que cuenta la biblioteca para dar alternativas es su sitio web, pues la comodidad de utilizar la red ya experimentada por los usuarios la ofrecen las webs bibliotecarias para acceder a la información y servicios que prestan. En el caso de la formación de usuarios ha llevado a los bibliotecarios a buscar nuevas prácticas y nuevos formatos. Un ejemplo son las biblioguías online objeto de este trabajo-, que presentan un formato electrónico, son accesibles desde cualquier lugar y favorecen un funcionamiento más autónomo por parte de los usuarios.

En nuestro estudio hemos podido comprobar que las bibliotecas universitarias españolas se encuentran en un estado de madurez en relación a la prestación de servicios y recursos de formación de usuarios en sus sedes webs. En un momento en el que este tipo de formación alcanza protagonismo por la necesidad, en el contexto de la Sociedad de la Información y del modelo universitario actual, de adquirir nuevas habilidades y competencias para desenvolverse con agilidad en la gestión de la información, también imprescindibles para funcionar en un mundo fundamentado por las telecomunicacio- nes y lo digital. Los contenidos de gran parte de las biblioguías recopiladas nos lo confirman.

Los datos analizados nos reflejan cómo la formación de usuarios ha evolucionado a un entorno más acorde con la alfabetización informacional que posibilita una concepción más individualizada y personalizada, pues gran parte de las variadas propuestas de guías elaboradas reflejan el propósito de facilitar que los usuarios adquieran unas competencias que ayuden a un desarrollo autónomo en los modelos de aprendizaje contemporáneos.

La formación en el entorno virtual nos parece que es una iniciativa adecuada para mantenerla y perfeccionarla con la finalidad de que siga siendo efectiva. En la medida que se diseñe con calidad e innovación se contribuirá a que se acuda a las sedes webs de las bibliotecas y que los usuarios las tengan como un referente imprescindible.

El panorama que reflejan los sitios webs analizados hace prever un futuro prometedor para las bibliotecas universitarias, en relación con las posibilidades que permite el espacio online para albergar estrategias y recursos de formación y aprendizaje.

Entendemos por biblioguías un tipo de guía elaborado por las bibliotecas universitarias y disponibles en sus sitios web para facilitar la formación de los usuarios en el uso de los recursos de información en general y en particular de los recursos y servicios más relevantes de la biblioteca. Para este tipo de guías también se utilizan otras denominaciones como guías de ayuda, tutoriales, material de formación o de autoformación, o simplemente guías.

Los bibliotecarios, con la elaboración de este tipo de recursos, en cierto modo ejercen de docentes o facilitadores del aprendizaje y esto nos lleva a preguntarnos cómo se preparan. Resolver este interrogante puede servir de planteamiento para otra vía de investigación.

También, este trabajo no has sembrado la inquietud de saber si los alumnos y docentes conocen la existencia de las secciones de biblioguías, y en qué medida las emplean y les son útiles. Para ello sería necesario ampliar esta investigación diseñando un estudio que tuviera como objetivos conocer el uso de estos recursos, y si existen estrategias de marketing por parte de los bibliotecarios para darlas a conocer.

\section{Apéndice}

Andalucía

Universidad de Almería

Nombre: Guías temáticas 
URL: http://cms.ual.es/UAL/universidad/serviciosgenerales/biblioteca/ servicios/FOR 24 GUIAS

Ubicación: Formación - Guias temáticas

Concepto: "Recursos de información disponibles en la Biblioteca, agrupados por áreas temática

Organización y clasificación: 9 materias

Formatos: $\mathrm{Html}$

Universidad de Córdoba

Nombre: Guías y tutoriales BUCO

URL: http://www.uco.es/servicios/biblioteca/formacion/ayestudio.html

Ubicación: Formación de usuarios - Guías y tutoriales BUCO Concepto:

Organización y clasificación: Guías en Slideshare (37), Guías en ISSUU (32), Guías y ayudas en pdf, ppt, prezi y html (15), Guías para elaborar un trabajo científico (3), Guías para la evaluación de la investigación (10), Otros (3)

Formatos: Pdf, ppt, prezi, html, Slideshare, ISSUU

Universidad de Granada

Nombre: Guías temáticas

URL: http://biblioteca.ugr.es/pages/biblioteca electronica/ guias tematicas

Ubicación: Biblioteca electrónica - Guías temáticas

Concepto: "Una ayuda que pretende organizar diferentes recursos

electrónicos, no en función del formato, soporte o medio de acceso,

sino agrupados por amplias áreas temáticas"

Organización y clasificación: 13 materias

Formatos: $\mathrm{Html}$

Universidad de Huelva

Nombre: Guías y ayuda

URL: http://guiasbuh.uhu.es/guias

Ubicación: Servicios - Guías y ayuda

Concepto: "Colección de recursos clasificados por temas que te

orientarán a la hora de buscar la información que necesitas"

Organización y clasificación: Guías por materias (9 materias), Ayuda

investigadores (6), Otras guías (2)

Formatos: $\mathrm{Html}$, vídeo

Universidad de Málaga

Nombre: Guías y tutoriales

URL: http://www.uma.es/ficha.php?id=74832

Ubicación: Ayudas y tutoriales - Guías y tutoriales

Concepto:

Organización y clasificación: Guía de nuestros servicios (1), Consulta al catálogo Jábega (1), Consulta a los recursos electrónicos (2). Acceso desde el exterior del campus (1), Renovación de material bibliográfico (1), Reserva de material bibliográfico (1), Mi historial de lecturas 81), Metabuscador (2), Guías de uso de bases de datos (3),

Peticiones de compras bibliográficas (PDI) (1)

Formatos: $\mathrm{Html}$

Universidad de Sevilla:

Nombre: Otras guías y ayudas

URL: http://guiasbus.us.es/c.php?g=129439\&p=845019

Ubicación: Estudia e investiga - Otras guías y ayudas

Concepto:

Organización y clasificación: Trabajos académicos (1), Conócenos

(2), Estudia e investiga (27), Otras guías de apoyo a la investigación Formatos: $\mathrm{Html}$

\section{Aragón}

Universidad de Zaragoza

Nombre: Guías de ayuda

URL: http://biblioteca.unizar.es/ayuda-y-formacion/guias-de-ayuda

Ubicación: Ayudas y formación - Guías de ayuda

Concepto: "La BUZ ha creado diverso material de ayuda para la

formación en sus recursos"

Organización y clasificación: Guías generales (4), Guías de recursos

(11), Para el aprendizaje y la investigación (6), Videotutoriales en la Biblioteca (5)

Formatos: Video, pdf, html, ppt

\section{Asturias}

Universidad de Oviedo

Nombre: Ayudas

URL: https://buo.uniovi.es/ayudas

Ubicación: Ayudas

Concepto: "Información que necesitas para utilizar nuestros recursos, herramientas y servicios (guías, tutoriales, manuales, etc.) y para desarrollar tu actividad académica a través de nuestros minisites que contienen fuentes de información para alumnos de grado, alumnos de postgrado, investigadores y diversas guías temáticas"

Organización y clasificación: Ayudas para utilizar bases de datos y plataformas (12), Ayudas para realizar búsquedas (7), Ayudas para utilizar gestores de citas y elaborar bibliografías (7), Ayudas a la producción científica (4), Otras ayudas (5), Trabajos fin de grado (1) Guías temáticas (23 materias)

Formatos: SlideShare, pdf, html

\section{Islas Baleares}

Universidad de Las Islas Baleares

Nombre: Ayuda

URL: http://biblioteca.uib.cat/oferta/ajuda/

Ubicación: Qué ofrecemos - ayuda

Concepto:

Organización y clasificación: Las bibliotecas y tu (16), Buscar infor-

mación (7) Cómo realizar un trabajo académico (2). Recursos para

la docencia y la investigación (4), Biblioayudas (15)

Formatos: Html, pdf, ppt,

Nombre: Guías temáticas

URL: http://biblioteca.uib.cat/oferta/recursos electronics/ guies tematiques/

Ubicación: Guías temáticas

Concepto: "Selecció de recursos d'informació en diferents àmbits temàtics de suport a la docència, l'aprenentatge i la investigació

Tenen com a objectiu ajudar i orientar en la recerca d'informació sobre una àrea temàtica concreta".

Organización y clasificación: 21 materias

Formatos: htm

\section{Canarias}

Universidad de La Laguna

Nombre: Guías y tutoriales

URL: https://www.ull.es/view/institucional/bbtk/Guias_y_tutoriales/es Ubicación: Guías y tutoriales

Concepto:

Organización y clasificación: PuntoQ (1 tutorial), Catálogo (3 tutoriales), Bases de Datos (19, 2 son tutoriales), Revistas electrónicas ( 9 , 5 son tutoriales), Libros electrónicos (4, 2 son tutoriales), Servicios

(8)

Formatos: Pdf, ppt, video

Universidad de Las Palmas de Gran Canaria

Nombre: Biblioguías

URL: http://biblioguias.ulpgc.es/

Ubicación: Formación - Biblioguías

Concepto:

Organización y clasificación: -Por materias:, Apoyo a la investigación

(14) , Apoyo al aprendizaje (7), Herramientas y servicios (13), Recursos electrónicos (39), Ciencias (12), Ciencias de la Salud (15),

Humanidades (26), Ingeniería y Arquitectura (14), Multidisciplinar (1), Sociales y jurídicas (23), -Por tipos:, Guía de uso general, Guía de materia

Formatos: $\mathrm{Html}$

\section{Cantabria}

Universidad de Cantabria

Nombre: Guías y tutoriales

URL: http://www.buc.unican.es/formacion

Ubicación: Docentes e investigadores - Personal docente e investigador - Cursos de formación. Guías y tutoriales

Concepto: "Guías, tutoriales y materiales formativos que cubren

diversos aspectos relacionados con el uso de la información científica"

Organización y clasificación: Sobre servicios de la biblioteca (11): catálogo, préstamo i, etc., Sobre recursos y técnicas de búsqueda (15), Sobre redacción de citas y referencias (13), Sobre gestores de bibliografía (11): EndNote, Mendeley, RefME, RefWorks, Zotero,

Sobre trabajos académicos (11), Sobre investigación y publicación científica (29)

Formatos: Html, pdf, prezi

\section{Castilla La Mancha}

Universidad de Castilla La Mancha

Nombre: Tutoriales de la biblioteca

URL: http://biblioteca.uclm.es/tutoriales.htm

Ubicación: Tu biblioteca - Tutoriales y videos

Concepto:

Organización y clasificación: Impacto y citas (4), Libros, revistas (2),

Gestores bi (4), Catálogo (1), Otros (presupuesto, Erasmus) (2)

Formatos: Video, pdf, ppt, html

\section{Castilla y León}

Universidad de Burgos

Nombre: Autoformación

URL: http://wwww.ubu.es/aprendizaje-e-investigacion/aulabiertaformacion-guias/autoformacion

Ubicación: Aprendizaje/investigación - aula@abierta: formación,

guías... - Autoformación

Concepto: La Biblioteca ofrece material de apoyo para facilitar el uso de los recursos de información a través del autoaprendizaje (elabo- 
ración de trabajos fin de grado y máster, gestión de referencias bibliográficas y citas, edición y producción científica...)

Organización y clasificación: Personal docente e investigador (7), Estudiantes de grado (2), Estudiantes de Máster, postgrado y doctorado (10)

Formatos: Pdf, html

Universidad de León

Nombre: Guías rápidas

URL: http://biblioteca.unileon.es/content/gu\%C3\%ADas-

r\%C3\%A1pidas

Ubicación: Aprendizaje e investigación - Guías rápidas

Concepto: "Intenta solventar necesidad de profesores y alumnos según dos cuestiones ¿Qué necesito? Y ¿Dónde lo busco?"

Organización y clasificación: PDI (25): sobre aspectos de la biblioteca, impacto, sexenios, libros y revistas, Alumnos (19): sobre aspectos de la biblioteca, búsqueda de información, Alumnos de primer año (16): sobre aspectos de la biblioteca, búsqueda de información Formatos: $\mathrm{Html}$

Universidad Pontificia de Salamanca

Nombre: Guías de autoformación

URL: https://www.upsa.es/biblioteca/servicio/tutoriales/

autoformacion.php

Ubicación: Cómo usar la biblioteca - Guias de autoformación

Concepto:

Organización y clasificación: Sobre Refworks (18), Sobre bases de datos (16), Sobre E-Libro (5), Sobre Realización, presentación, difusión y evaluación de trabajos académicos y científicos (16), Sobre Summa (3)

Formatos: Pdf

Universidad de Valladolid

Nombre: Guías rápidas

URL: http://biblioteca.uva.es/export/sites/biblioteca/3.servicios/3.09.la

bibliotecaconlainvestigacion/3.09.03.guiasrapidas/index.html

Ubicación: Servicios - Biblioteca con la investigación - Guías rápidas

Concepto:

Organización y clasificación: En fase de actualización

Formatos: En fase de actualización

\section{Cataluña}

Universidad Autónoma de Barcelona

Nombre: Guías de las bibliotecas de la UAB

URL: http://ddd.uab.cat/collection/guibib? In=es

Ubicación: Servicios - Apoyo a la docencia e investigación - Guías de las bibliotecas de la UAB

Concepto: "Engloba todo tipo de guías "enfocadas al conocimiento de las herramientas para la investigación, las bases de datos y los recursos temáticos que la UAB pone a vuestra disposición"

Organización y clasificación: Bases de datos (11), Catálogo (12),

Cómo encontrar (7), Recursos temáticos (15), Servicios (20), Guías

de las bibliotecas

Formatos: $\mathrm{Pd}$

Universidad de Lérida

Nombre: BiblioGuías

URL: http://biblioguies.udl.cat/friendly.php?s=guies

Ubicación: BiblioGuías

Concepto: "Selecció de recursos d'informació vinculats amb l'aprenentatge, la docència i la recerca. Contenen recursos web rellevants $i$ l'accés als recursos electrònics més significatius per a cada àmbit de coneixement"

Organización y clasificación: Guías temáticas (16), Guías de exposiciones (4), Guías de soporte a la investigación (5), Guías genéricas (2)

Formatos: $\mathrm{Html}$

Universidad Pompeu Fabra

Nombre: Guías y tutoriales

URL: https://www.upf.edu/bibtic/guiesiajudes/

Ubicación: Biblioteca - Guías y tutoriales

Concepto:

Organización y clasificación: La biblioteca y las TIC (8), Guías de uso del Aula Global (2), Derechos de autor (5), Herramientas para el aprendizaje y la investigación (13), Recursos audiovisuales (7), Protocolo de entrada en datos personales (6), Todas las Guías BibTIC, Guías temáticas

Formatos: Pdf, video, html

Universidad Rovira i Virgili

Nombre: Guías y tutoriale

URL: http://www.urv.cat/ca/vida-campus/serveis/crai/que-us-

oferim/formacio-competencies-nuclears/autoformacio/

Ubicación: Soporte a la formación - Guías y tutoriales

Concepto: "Materials d'autoaprenentatge amb la finalitat que pugueu aprendre de manera autònoma el funcionament del catàleg, de les bases de dades, dels serveis o dels recursos d'informació que posem al vostre abast des del CRAl"
Organización y clasificación: Tripticos (4), Guías de catálogos (4), Guías de servicios (6), Guías de los recursos electrónicos del CRAI (74), Tutoriales (5)

Formatos: ISSUO, video

Comunidad de Madrid

Universidad de Alcalá de Henares

Nombre: Guías temáticas

URL: http://wikibuah.pbworks.com/w/page/65722818/Inicio-

Gu\%C3\%83\%C2\%ADas

Ubicación: Recursos de información - Guías temáticas

Concepto: "La Biblioteca estructura por materias la información, lo que facilita conocer sus recursos, dónde y cómo localizarlos, e incluye una selección de recursos en Internet que pueden ser de interés para el aprendizaje, la docencia y la investigación a los usuarios de la Universidad de Alcalá., La selección de recursos está en constante revisión y actualización"

Organización y clasificación: 18 materias

Formatos: Html

Nombre: Ayuda

URL: http://biblioteca.uah.es/servicios/accesibilidad.asp

Ubicación: Ayuda

Concepto:

Organización y clasificación: Guía buscador, Guía catálogo, Guía

e Buah, Autoformación, Videotutoriales

Formatos: $\mathrm{Html}$

Universidad Autónoma de Madrid

Nombre: Biblioguías de la Biblioteca

URL: http://biblioguias.uam.es/c.php?g=235585

Ubicación: Qué ofrecemos - Guías y tutoriales - Biblioguías

Concepto: "Guías, tutoriales y recursos de apoyo para la investiga-

ción y la docencia"

Organización y clasificación: Guias por bibliotecas (8 bibliotecas) Tutoriales y recursose-e (orden alfabético), Apoyo al aprendizaje (especie de gráfico con enlaces), Apoyo a la investigación (especie de gráfico con enlaces)

Formatos: $\mathrm{Html}$, pdf, video

Universidad Carlos III

Nombre: Tutoriales y guías

URL: http://www.uc3m.es/ss/Satellite/Biblioteca/es/TextoMixta/13712

12366749/UTEID_Unidad_de_Tecnologia_Educativa_e_Innovacion_ Docente

Ubicación: Servicios - Apoyo a la innovación docente -Tutoriales y

guías

Concepto:

Organización y clasificación: Guías para la preparación, impartición y seguimiento de MOOCs (5), Guía para creación de videos educativos

(1), Videotuoriales sobre creación de videotutoriales (2)

Formatos: Pdf, html, video

Nombre: Guías temáticas

URL: http://uc3m.libguides.com/guias_tematicas

Ubicación: Encontrar información - Guías temáticas

Concepto:

Organización y clasificación: 10 materias

Formatos: $\mathrm{Html}$

Universidad Complutense de Madrid

Nombre: Autoformación

URL: https://biblioteca.ucm.es/autoformacion

Ubicación: Servicios - formación virtual - autoformación

Concepto:

Organización y clasificación: Cursos sobre información (9), Cursos

de la BUC (6), Otros (5), Videos y tutoriales (7)

Formatos: Html, pdf, video

Universidad Pontificia de Comillas

Nombre: Guias y formación

URL: http://www.comillas.edu/es/biblioteca/guias-y-tutoriales

28.Ubicación: Guías y formación

Concepto:

Organización y clasificación: Guía para encontrar formación, Elaboración de trabajos científicos, Cómo citar, Guía sobre acceso abierto Formatos: Html, pdf

Universidad Rey Juan Carlos

Nombre: Guías y tutoriales

URL: http://www.urjc.es/estudiar-en-la-urjc/biblioteca/400-formacion

Ubicación: Formación - Guías y tutoriales

Concepto:

Organización y clasificación: Servicios y herramientas Biblioteca

URJC (9)

Formatos: video

UNED

Nombre: Guías y tutoriales

URL: http://portal.uned.es/portal/page? pageid=93,26044627\& dad =portal\&_schema=PORTAL 
Ubicación: Aprendizaje e investigación - Guías y tutoriales Concepto:

Organización y clasificación: Guías, Guías de uso (6), Guías rápidas 5' (20), Guías sobre Linceo+ (1), Guía de herramientas de análisis de la actividad investigadora (1), Guías de investigación por materias

(18), Guía sobre uso ético de la información y citas bibliográficas (1) Guías BibUNED (36), Tutoriales, Utilizar la biblioteca (21), Competencias en información (32), Competencias digitales básicas (17), Recursos de ciencias (0), Recursos de ciencias sociales y humanidades (9), Apoyo al PDI (acreditaciones,etc.) (35)

Formatos: Video, html, pdf

\section{Comunidad Valenciana}

Universidad de Alicante

Nombre: Guías temáticas

URL: https://biblioteca.ua.es/es/encuentra-informacion/guiastematicas/guias-tematicas.html

Ubicación: Encuentra información - Guías temáticas

Concepto:

Organización y clasificación: 11 materias

Formatos: $\mathrm{Html}$

Universidad Jaime /

Nombre: Material de formación

URL: http://www.uji.es/serveis/cd/bib/serveis/investigacio/

materialformacio/

Ubicación: Servicios - Apoyo a la investigación científica - Materia de formación

Concepto:

Organización y clasificación: Identidad digital y perfil investigador,

Fuentes de información para investigadores, El Repositorio de la UJI Competencias informacionales: búsqueda y recuperación de la

información para la investigación, Competencias informacionales en

el ámbito de la investigación, Data Management Plan and Socia

Impact of Research

Formatos: Prezi, html, video, SlideShare

Nombre: Guías y manuales

URL: http://repositori.uji.es/xmlui/handle/10234/10776

Ubicación: Información - Normativas y documentos de la biblioteca Guías y manuales

Concepto:

Organización y clasificación: Manual de uso y buenas prácticas de las Redes Sociales de la Biblioteca Jaume I, Manual de gestió de l'Arxiu General, Guía de la biblioteca, Manual de documents i llenguatge administratiu, ALFIN: Accés als recursos d'informació bibliográfica i documental de la Universitat Jaume I

Formatos: Pdf

Universidad Politécnica de Valencia

Nombre: Biblioguías

URL: http://biblioguias.webs.upv.es/bg/index.php/es/

Ubicación: Usar la biblioteca - Préstamo interuniversitario - Bibloguías

Concepto: "Guías y ayudas donde encontrarás diferentes recursos elaborados o recopilados por las Bibliotecas UPV, organizados de manera temática y que pueden ser de tu interés"

Organización y clasificación: Conoce la biblioteca (5), Cómo buscar información (26), Factor de impacto y citas recibidas (15), Citas y referencias bibliográficas (35), Recursos para TFG/TFM (20), Propiedad intelectual (18), Guías temáticas (17)

Formatos: Html, pdf, video

\section{Extremadura}

Universidad de Extremadura

Nombre: Biblioguías

URL: http://biblioguias.unex.es/

Ubicación: Utilizar - Biblioguías

Concepto:

Organización y clasificación: Guías para el aprendizaje (11) Guías para la investigación (11), Guías temáticas (32), Herramientas para buscar y usar la información (6)

Formatos: Html, video

\section{Galicia}

Universidad de La Coruña

Nombre: Guías

URL: http://www.udc.es/biblioteca/servizos/apoio investigacion/ recursos_info/index2.html

Ubicación: Soporte a la investigación y al aprendizaje - Recursos de información - Guías

Concepto:

Organización y clasificación: Guías de recursos electrónicos (78),

Guías de herramientas de trabajo (9)

Formatos: Html, pdf

Universidad de Santiago de Compostela
Nombre: Guías

URL: http://sp.bugalicia.org/san/subjects/index.php

37.Ubicación: Recursos electrónicos - guías

Concepto:

Organización y clasificación: Temáticas (0), Apoyo al estudio y a la

investigación (11)

Formatos: $\mathrm{Html}$

Universidad de Vigo

Nombre: Guías y tutoriales

URL: http://sp. bugalicia.org/vig/subjects/index.php

Ubicación: Servicios - Guías y tutoriales

Concepto:

Organización y clasificación: Apoyo a la investigación (14), Citar y gestionar una bibliografía (12), Otras guías (7), Materias (27, pero se incluyen parte de las anteriores)

Formatos: $\mathrm{Html}$

\section{Navarra}

Universidad de Navarra

Nombre: Recursos por materias y guías

URL: http://apps-bibl.si.unav.es/sp/subjects/index.php

Ubicación: Investigación y aprendizaje - Recursos por materias y

guías

Concepto: "Guías elaboradas por los bibliotecarios temáticos con los

recursos más interesantes en cada área"

Organización y clasificación: Por materias en orden alfabético

muchas, etc

Formatos: $\mathrm{Html}$

Universidad Pública de Navarra

Nombre: Guías temáticas

URL: http://guias-tematicas.unavarra.es/sp/subjects/index.php

Ubicación: Colecciones y recursos - Guías temáticas

Concepto:

Organización y clasificación: Por materias en orden alfabético,

muchas, etc

Formatos: Htm

\section{País Vasco}

Universidad de Deusto

Nombre: Biblioguías

URL: http://biblioguias.biblioteca.deusto.es/

Ubicación: Aprendizaje e investigación - Biblioguías

Concepto: "Te orientan sobre los recursos y servicios más relevantes de la biblioteca"

Organización y clasificación: Guías de apoyo al aprendizaje e investigación (14), Guías de uso de base de datos (12), Guías de uso de servicios (7), Guías temáticas (15), Valoración de la actividad investigadora (12)

Formatos: $\mathrm{Htm}$

\section{Región de Murcia}

Universidad Católica San Antonio

Nombre: Videos formación biblioteca

URL: http://biblioteca.ucam.edu/servicios/gabinete-formacion/videosformacion/formacion

Ubicación: Cursos de formación - Videos formación biblioteca

Concepto:

Organización y clasificación: 4 videos

Formatos: Video

Universidad de Murcia

Nombre: Autoformación

URL: http://www.um.es/web/biblioteca/contenido/formacion/ autoformacion

Ubicación: Formación de usuarios - Autoformación

Concepto: "Guías de uso de los recursos más utilizados. En algunos casos son tutoriales que ofrecen los creadores de los recursos de

información. Otras son guías y presentaciones realizadas por bibliotecarios de la Universidad de Murcia, utilizadas en nuestras sesiones de formación"

Organización y clasificación: Catálogo Alba (7) , Formación básica

(6), Formación especializada (7), Recursos online (externos) (5)

Formatos: Pdf, video

Universidad Politécnica de Cartagena

Nombre: Guías y manuales

URL: http://www.bib.upct.es/guias-manuales

Ubicación: Tutoriales - Guías y manuales

Concepto:

Organización y clasificación: Buscador UPCT (1), Repositorio Digital

UPCT (1)

Formatos: Pdf 


\section{Referencias}

Anexo: Universidades de España. // Wikipedia. https://es.wiki pedia.org/wiki/Anexo:Universidades_de_Espa\%C3\%B1a (2017-01-12).

Anglada, Lluís (2012). Bibliotecas universitarias: cabalgando la tecnología, siguiendo al usuario. // El profesional de la informaión. 21:6 (Noviembre-Diciembre 2012) 553-556.

Bracke, Paul J.; Dickstein, Ruth (2002). Web tutorials and scalable instruction: testing the waters. // Reference Services Review. 30:4 (2002) 330-337.

Detmering, Robert; Johnson, Anna Marie; Sproles, Claudene; McClellan, Samantha; Hernandez Linares, Rosalinda (2015). Library instruction and information literacy 2014. // Reference Services Review. 43:4 (2015) 533-642.

Dewald, Nancy H. (1999). Transporting good library instruction practices into the web environment: an analysis of online tutorials. // The Journal of Academic Librarianship. 25:1 (1999) 26-32.

Fernández-Ramos, Andrés (2015). Alfabetización informativa a través de tutoriales electrónicos: nuevo reto para las bibliotecas universitarias. // La información y sus contextos en el cambio social. UNAM: Instituto de Investigaciones Bibliotecológicas y de la Información, 2015.127-153.

Fernández-Ramos, Andrés (2016). Online library tutorials in Mexican universities: presence and characteristics. // Library Hi Tech. 34:4 (2016) 787-802.

Giappiconi, Thierry; Pirsich, Volker; Hapel, Rolf (2001). Servicios de futuro basados en Internet en las bibliotecas públicas. Una investigación con ejemplos. Barcelona: Fundación Bertelsmann, 2001.

Gómez Hernández, José Antonio (2005). Alfabetización informacional: cuestiones básicas. Nota informativa enviada a Thinkepi (6 de junio de 2005) http://thinkepi.net/repositorio/alfabetizacioninformacional -cuestiones-basicas/. (2017-03-12).

Gómez Hernández, José Antonio (2010). Las bibliotecas universitarias y el desarrollo de las competencias informacionales en los profesores y los estudiantes. // Revista de Universidad y Sociedad del Conocimiento (RUSC). 7:2 (2010) 39-49. http://rusc.uoc.edu/ojs/index.php/rusc/ article/view/v7n2-gomez/v7n2-gomez. (2017-03-10).

Gómez Hernández, José Antonio; Licea de Arenas, Judith (2002). La alfabetización en información en las Universidades. // Revista de Investigación Educativa. 20:2 (2002) 469-486

Gómez Hernández, José Antonio; Benito Morales, Félix (2001). De la formación de usuarios a la alfabetización informacional. // SCIRE. 7:2 (2001) 53-83. http://www. um.es/gtiweb/jgomez/publicaciones/alfabinforzaragoza2. PDF. (2017-03-01).

Iribarren-Maestro, Isabel; Grandal, Teresa; Alecha, María; Nieva, Ana; San-Julián, Teresa (2015). Apoyando la investigación: nuevos roles en el servicio de bibliotecas de la Universidad de Navarra. // El profesional de la información. 24:2 (2015) 131-137.

López Zazo, Rhut (2007). Formación de usuarios y alfabetización informacional en las bibliotecas universitarias: propuesta de planificación para un CRAI. https://pendiente demigracion.ucm.es/BUCM/biblioteca/doc10057.pdf (2017-03-19)

Martínez Ballester, Inmaculada (2014). Formación de usuarios online en bibliotecas universitarias: estado de la cuestión. // Cuadernos de Gestión de Información. 4 (2014) 38-50
Ministerio de Cultura (2017). Directorio de bibliotecas españolas. http://directoriobibliotecas.mcu.es/portada.html (2017-02-14).

Pérez Hidalgo, Anna; Méndez Martínez, Maribel (2000). La formación de usuarios en una biblioteca virtual universitaria. Biblioteca virtual de la UOC. http://openaccess.uoc.edu/webapps/o2/bitstream/10609/ 348/1/3499.pdf. (2017-03-12).

Pinto, María; Sales, Dora; Osorio, Pilar (2008). Biblioteca universitaria, CRAI y alfabetización informacional. Gijón: Ediciones TREA, 2008.

Reynolds, Latisha; McClellan, Samantha; Finley, Susan; Martinez, George; Hernandez Linares, Rosalinda (2016). Library instruction and information literacy 2015. // Reference Services Review. 44:4 (2016) 436-543.

Rutter, Lynne; Matthews, Marian (2002). InfoSkills: a holistic approach to on-line user education. // The Electronic Library. 20:1 (2002) 29-34

Sacchanand, Chutima; Jaroenpuntaruk, Vipa (2006). Development of a web-based self-training package for information retrieval using the distance education approach. // The Electronic Library. 24:4 (2006) 501-516.

Serrano Vicente, Rocío (2005). Alfabetización en información en bibliotecas universitarias. Algunas experiencias en el ámbito anglosajón. // $9^{a}$ Jornadas Españolas de Documentación (2005). Madrid: FESABID, 2005. 509-520.

Somoza Fernández, Marta (2003). Tutoriales temáticos interactivos: nuevos modelos para nuevos entornos didácticos. // VIII Jornadas Españolas de Documentación (Barcelona 2003). Barcelona: FESABID, 2003. 477-484.

Somoza-Fernández, Marta; Abadal, Ernest (2007). La formación de usuarios en las bibliotecas universitarias españolas. // El profesional de la información. 16:4 (JulioAgosto 2007) 287-293.

Somoza-Fernández, Marta; Rodríguez-Parada, Concepción (2011). Tutoriales universitarios: indicadores y ejemplos de buenas prácticas. // El profesional de la información. 20:1 (enero-febrero 2011) 38-46.

Tobin, Tess; Kesselman, Martin (1999). Evaluation of webbased library instruction programs. // 65th IFLA Council and General Conference (Bangkok, Tailandia, 20-28 agosto 1999). http://www.ifla.org/IV/ifla65/papers/102163e.htm. (2017-03-12).

Tovar-Sanz, María R. (2015). El apoyo a la investigación en las bibliotecas universitarias españolas. // Documentación de las Ciencias de la Información. 38 (2015) 311326.

Warnken, Paula (2004). The impact of technology on information literacy education in libraries. // The Journal of Academic Librarianship. 30:2 (2004) 151-156.

Wickramanayake, Lalith (2012). Instruction and help services in the academic library websites and web pages in Sri Lanka: A content analysis. // The Electronic Library. 30:3 (2012) 377-389.

Yi, Hua (2005). Library instruction goes online: an inevitable trend. // Library Review. 54:1(2005) 47-58.

Zhang, Tao; Stonebraker, Llana; Promann, Marlen (2016). Understanding library users' preferences and expectations of online help. // Reference Services Review. 44:3 (2016) 362-374.

Enviado: 2017-03-24. Segunda versión: 2017-05-31 Aceptado: 2017-09-12. 